meteor are low, they are missing from radar surveys that do not reach below $10^{10}$ electrons $\mathrm{m}^{-1}$.

The Havana-Sidell simultaneous radar-television observations determined ionizing probabilities for meteors with photographic absolute magnitudes +4 to +8 , velocities $14-36 \mathrm{~km} \mathrm{~s}^{-1}$, and heights 82-101 km (Cook et al., Coll., 13). As stated above, fragmenting meteors were omitted from the analysis. Observed ionizing probabilities for other magnitudes and velocities are, of course, much to be desired. It will be necessary but difficult to eliminate the effects of dissociative recombination from ionizing probabilities derived from radar-visual and radar-photographic observations.

The 'initial radius' of the ionized column (the radius after a millisecond or so) has been measured using observations at two or three wavelengths $(03.104 .020 ; 06.104 .030 ; 06.104 .011)$. Contrary to simplified physical theories, the initial radius was found to be approximately proportional to the 0.5 power of the atmospheric meanfree path. Fragmentation, however, needs to be taken into account in the analysis of initial-radius data. The upper bound to heights observed at Havana appeared to be diffusion rather than initial radius (Southworth, Coll., 13); this suggests that initial radius may depend on magnitude. At Kiev (06.104.010), the radial distribution of electrons in the ionized column was observed to fall off slightly faster, in the mean, than in a Gaussian distribution.

Deceleration of radar meteors was observed at Kharkov $(03.104 .051 ; 04.104 .036)$ and at Havana. The ionization curve has been determined statistically at Kharkov (06.104.109) and has been routinely observed at Havana.

Electron attachment rates have been observed in enduring echoes at Dushanbe (05.104.021; 06.104.063), as well as a semidiurnal variation in the attachment rate (06.104.049). A diurnal variation in the attachment rate below $90 \mathrm{~km}$ has been predicted on aeronomic grounds (Baggaley, 1972).

Any agreement on the distribution of orbital elements of radar meteors must await agreement on the physical selection effect. No distribution corrected for all defects seems to have been published. However, the distribution of orbits observed in Havana from October 1968 to December 1969 has been corrected to yield the orbital distribution of meteors of equal mass, using the ionizing probability determined there. It is possible, however, that there is still a selection effect against fragmenting meteors. This corrected distribution contains less than $1 \%$ retrograde meteors and mostly with inclinations under $20^{\circ}$; the majority of aphelia are $<3 \mathrm{AU}$ and the majority of perihelia $<0.7 \mathrm{AU}$; the mean velocity outside the Earth's atmosphere is under $20 \mathrm{~km} \mathrm{~s}^{-1}$.

\title{
REFERENCE
}

Baggaley, W. J. $\quad$ 1972, Monthly Notices Roy. Astron. Soc., 159, 203.

\section{METEOR SPECTROSCOPY}

\section{P. M. Millman}

Programs of meteor spectroscopy have been continued vigorously in the U.S.A., Canada, Czechoslovakia, and the U.S.S.R. In particular, in North America, the use of batteries of Maksutovtype optical systems equipped with shutters triggered by photomultipliers, and the employment of image-intensifying electronic devices, have resulted in the recording of large numbers of faintmeteor spectra in the 2- to 5-mag. range (05.104.042, 3; Harvey, Coll. 13). These, together with the records of the faint upper portions of bright-meteor trajectories, show promise of giving important informations about the contribution of the lines and the band systems of the lightweight elements hydrogen, carbon, nitrogen, and oxygen to the visible radiation of meteors. In this connection, further improvement of electronic image-intensifier systems, together with the use of these devices in spectrophotometry, is a pressing need for the immediate future. The possibility of extending these investigations into the near ultraviolet and the near infrared should not be overlooked.

No less important is the detailed photometry of faint features in the many-lined spectra of very brilliant fireballs. Research in Czechoslovakia has demonstrated that the band structures of lightweight molecules can be detected in the background among the bright atomic lines, if the dispersion is high enough (06.104.039). Further work in this field should be encouraged as it will contribute 
additional data on the role played by the elements of low atomic number in the structure of cometary meteroids.

Most meteor spectra secured are of cometary meteroids. Since little is known of the quantitative chemical composition of cometary material, an important problem for the future is how to use meteor spectra to derive chemical compositions of meteroids. The temperature concept, based on considerations of thermodynamical equilibrium, is unreliable for quantitative estimates. Thanks to the recent laboratory determinations of atomic-collision cross sections under conditions that simulate meteor radiation (04.104.012; 06.104.002, 3; Boitnott and Savage, 1972), a start has been made in calculating meteroid compositions (Millman, 1972). But we badly need more laboratory values for the relevant atomic constants, and more good photometry of detailed meteor spectra. Eventually this should lead us to a study of the differences in composition among the meteroids originating from various comets.

Some theoretical work has been attempted in an effort to explain the presence or absence of certain enduring features in meteor spectra, such as that near $3840 \AA$ and the line of neutral oxygen at $5577 \AA$. So far, this effort has been very successful and much remains to be done on the theory, not only of the enduring features, but also on the relation between the ionized lines and the neutral lines and on the relative strengths of the band systems of the light elements in meteor spectra. Note should be taken of the current activity concerned with the theoretical study of molecules in the coma of a comet. Since the meteor vapor originates from solids originally imbedded in the type of solid that produces the comet vapor, progress on research in the two fields should be closely coordinated.

Another important field of investigation that should be considered in this problem of the chemical composition of cometary matter is the mass-spectrometer measures made in the ionosphere with the aid of upper-air rockets (Narcisi, 1971; Goldberg and Aikin, 1971). Interpretation of these measures is bedeviled by the large number of chemical and photochemical processes that establish the measured concentrations of ions. A large amount of data in this field have been accumulated recently, both in North America and in Europe, and it seems highly probable that most of the metallic ions detected are a fallout from meteroids. The frequency of the elements recorded should be correlated with results from meteor spectroscopy, and further correlated with the compositions found for particles of presumed interplanetary dust collected by devices mounted on satellites, rockets, and balloons.

In the various types of observational programs referred to above, the preliminary work on meteoroid composition suggests some affinity between the meteoroids and the carbonaceous chondrites. The big question still remains - what is the quantitative contribution of the lightweight elements and molecules to this picture?

\section{REFERENCES}

Boitnott, C. A., Savage, H. F. $\quad$ 1972, Astrophys. Journ., 172, 201.

Goldberg, R. A., Aiken, A. C. $\quad$ 1971, Journ. Geophys. Res., 76, 8352.

Millman, P. M. 1972, Journ. Roy. Astron. Soc. Canada, 66, 201.

Narcisi, R. S. 1971, in F. Verniani, (ed.), Upper Atmosphere Physics, Casa Editrice Compositori, Bologna, 12.

\section{PHYSICAL THEORY OF METEORS}

\section{A. F. Cook}

Development of a viable physical theory of meteors requires a suitable underpinning of observations to keep within reasonable bounds the proliferation of possible detailed processes considered. Optical observations are subject to much more straightforward interpretation than are radar observations, and the smaller the meteoroid the more simple we may hope to find the physical conditions. These considerations lead to the urgent importance of observations of the faintest possible meteors, both with and without dispersion. The best techniques, at present, seem to be those of image intensification and low-light-level television. Other supporting observations are required, 